\title{
FORMACIÓN DE TRADUCTORES E INTÉRPRETES. AVANZANDO EN EL ENFOQUE POR COMPETENCIAS
}

Anabel Galán-Mañas (https://orcid.org/0000-0002-5498-9171)

Departamento de Traducción e Interpretación y Estudios de Asia Oriental, Universitat Autònoma de Barcelona

L interés sobre la adquisición de la competencia traductora ha crecido en las últimas décadas y ha ido evolucionado en función de las corrientes pedagógicas del momento. Si bien las primeras propuestas didácticas para la formación de traductores e intérpretes se centraban en los estudios contrastivos, con el tiempo se le ha otorgado mayor importancia al proceso. En esta línea surgieron propuestas basadas en objetivos de aprendizaje (Delisle, 1980, 1993) y, más adelante, con la adopción de un enfoque constructivista (Kiraly, 2000), aparecieron propuestas centradas en las tareas de traducción (Hurtado, 1996, 1999; González Davies, 2003, 2004) o en la formación por competencias (Kelly, 2005; Hurtado, 2007, 2015).

Este número especial sobre pedagogía y didáctica de la traducción y la interpretación incluye investigaciones y experiencias docentes que tienen como objetivo indagar sobre la efectividad de ciertas metodologías en la formación de traductores e intérpretes. Todas ellas sitúan al alumnado en el centro de la formación y fomentan el aprendizaje significativo, activo y reflexivo con la finalidad de preparar a traductores e intérpretes competentes. El monográfico contiene 14 artículos, de los cuales seis son artículos de investigación, dos son revisiones y seis son experiencias docentes.

En el primer artículo Mohammad Reza Esfandiari, de Islamic Azad University, Helia Vaezian, de Khatam University, y Forough Rahimi, de la Shahid Behehsti University of Medical Sciences, analizan la compatibilidad del marco de referencia del Máster Europeo en Traducción (EMT, en sus siglas en inglés) con las necesidades de los traductores no europeos. Los autores constataron que los 
traductores profesionales difieren ligeramente de las competencias que prioriza el EMT y otorgan mayor importancia a las competencias lingüística, temática e intercultural.

Ana Gregorio Cano, de la University of Texas at Arlington, presenta una investigación sobre el desarrollo de la competencia estratégica. En concreto, la autora conduce un estudio longitudinal para analizar el metalenguaje utilizado por los estudiantes de Traducción e Interpretación en la identificación y descripción de problemas de traducción, tanto al inicio como al final de su formación.

El tercer artículo viene de la mano de María del Mar Haro Soler, de la Universidad de Granada. Su trabajo estudia el efecto en el estudiantado de prácticas como el pensamiento autorreferente, la persuasión verbal, el aprendizaje vicario o el establecimiento de objetivos en la autoeficacia para traducir. Según la autora, estas prácticas, que promueven el aprendizaje activo y centrado en el estudiante, deberían generalizarse en la formación de traductores.

Laura Cruz García, de la Universidad de Las Palmas de Gran Canaria, presenta un interesante estudio sobre la especificidad de la enseñanza de lenguas para traductores e intérpretes. La autora describe cómo se explotan los textos en las clases de lengua extranjera en 13 facultades españolas de Traducción e Interpretación.

En el quinto artículo, Cinta Gallent Torres e Isabel Tello Fons, ambas de la Universidad Internacional de Valencia, resaltan la necesidad de formación en la competencia informacional para evitar que el estudiantado cometa ciberplagio. Las autoras constatan en su estudio que los estudiantes cometen ciberplagio por desconocimiento, porque no saben cómo y cuándo referenciar las fuentes bibliográficas, así como de las sanciones que podrían derivarse.

Una investigación sobre la traducción de aplicaciones móviles en la asignatura de localización constituye el sexto artículo. Su autora, María del Mar Sánchez Ramos, de la Universidad de Alcalá, defiende que, aunque este tipo de localización comparte características con la localización de programas informáticos, es necesario incluirla en la formación de traductores por la dificultad que encuentran los estudiantes en la resolución de aspectos lingüísticos, técnicos y culturales específicos de las aplicaciones móviles. 
Elisa Calvo, de la Universidad Pablo de Olavide, presenta una propuesta de formación situacional para el aula de traducción publicitaria. Se trata de un modelo que integra el aprendizaje por proyectos y los portafolios y que permite a los alumnos ejercer diferentes roles y desarrollar distintas tareas propias de la traducción profesional.

Elena Rodríguez-Murphy, de la Universidad de Salamanca, a partir del análisis de las principales dificultades de los estudiantes en la traducción inversa, en concreto al inglés, constata que el principal problema a que tienen que hacer frente es el uso de preposiciones y otras partículas, y propone una serie de actividades formativas para superar dicha dificultad.

Entre las experiencias docentes encontramos la de Ángela Patricia Morales Santibáñez, de la Universidad de Santiago de Chile. La autora describe un proyecto de traducción para desarrollar la competencia temática en el ámbito de las ciencias sociales. Los estudiantes trabajan de forma colaborativa para resolver un encargo en el que deben traducir textos auténticos simulando una situación de trabajo real.

Romina Valeria Carabajal, de la Universidad Nacional del Comahue, propone el trabajo en forma de talleres en el aula de traducción técnica. El objetivo es implementar las metodologías activas en un contexto tradicionalmente transmisionista. La autora considera que la realización de tareas de traducción de forma colaborativa por parte de los alumnos integra la teoría en actividades eminentemente prácticas.

Otra de las experiencias docentes nos llega de la mano de Raquel Sanz-Moreno, de la Universidad de Valencia, y consiste en contar con un profesional del ámbito médico-sanitario en las clases de interpretación en los servicios públicos. De la experiencia se desprende el carácter activo que adoptan las clases, así como la adquisición de las destrezas interpretativas y temáticas por parte del alumnado.

Cristina Álvarez de Morales Mercado, de la Universidad de Granada, presenta una propuesta didáctica de corte constructivista para el aula de traducción accesible que se centra en la formación de la audiodescripción en entornos turísticos. Para ello los estudiantes tienen que crear, de forma autónoma y colaborativa, materiales accesibles de dos monumentos arquitectónicos y superar los límites físicos del aula. 
Francisco J. Vigier Moreno, de la Universidad Pablo de Olavide, presenta una experiencia docente para la enseñanza de la traducción jurídica del alemán al español centrándose en el género citación judicial. El autor propone la simulación de un proyecto de traducción en que los alumnos deben hacer un ejercicio de textología y derecho comparado, y tomar las decisiones correspondientes. La propuesta favorece la toma de conciencia del proceso de adquisición, desarrollo y consolidación en traducción jurídica por parte del propio estudiante.

Finalmente, frente al auge de la formación de traductores a distancia, Diego Mansilla, de la University of Massachusetts, y María González-Davies, de la Universidad Ramon Llull, proponen una interesante experiencia didáctica que se centra en el uso explícito de estrategias de aprendizaje sociafectivas en un curso virtual de traducción. Este tipo de estrategias, basadas en el aprendizaje colaborativo, tienen como finalidad compensar la falta de presencia física de los aprendices.

\section{REFERENCIAS}

Delisle, Jean (1980). L'analyse du discours comme méthode de traduction. Ottawa: Editions de l'Université d'Ottawa.

Delisle, Jean (1993). La Traduction raisonnée : manuel d'initiation à la traduction professionnelle de l'anglais vers le français. Méthode par objectifs d'apprentissage. Otawa: Les Presses de l'Université d'Ottawa.

González Davies, M. (2003). Secuencias. Tareas para el aprendizaje interactivo de la traducción especializada. Barcelona: Octaedro.

González Davies, M. (2004). Multiple Voices in the Translation Classroom. Activities, Tasks and Projects. Amsterdam y Filadelfia: John Benjamins.

Hurtado, A. (1996). La enseñanza de la traducción directa general. Objetivos de aprendizaje y metodología. En A. Hurtado (Ed.). La enseñanza de la traducción (pp. xx-xx). Castellón de la Plan:. Servei de Publicacions de la Universitat Jaume I.

Hurtado, A. (1999). Enseñar a traducir, Metodología en la formación de traductores e intérpretes. Madrid: Edelsa.

Hurtado, A. (2007). Competence-Based Curriculum Design for Training Translators. The Interpreter and Translator Trainer, 1(2), 163195. doi: https://doi.org/10.1080/1750399X.2007.10798757

Hurtado, A. (2015). Aprender a traducir del francés al español: competencias y tareas para la iniciación a la traducción. Castellón de la Plana: Servei de Publicacions de la Universitat Jaume I.

Kiraly, D. (2000). A Social Constructivist Approach to Translator Education; Empowerment from Theory to Practice. Manchester y Northampton: St. Jerome Publishing 